\title{
Higher Dimensional Topological Defect Solutions with Quark Matter in Gravitation Theories
}

\author{
Halife Cag̃ lar ${ }^{*}$ and Sezgin Aygün ${ }^{2}$ \\ ${ }^{1}$ Institute for Natural and Applied Sciences, Çanakkale Onsekiz Mart University, 17020, Çanakkale, Turkey \\ ${ }^{2}$ Çanakkale Onsekiz Mart University, Arts and Sciences Faculty, Department of Physics, Terziog̃lu Campus 17020, \\ Turkey \\ Email: hlfcglr@gmail.com
}

\begin{abstract}
In this paper we have researched higher dimensional flat Friedmann-Robertson-Walker (FRW) cosmological model solutions for string cloud and domain walls coupled with quark and strange quark matters in Lyra and Riemann geometries. We have obtained that string tension density is zero in Lyra and Riemann geometries. Also, the displacement field vector behaves like a cosmological constant in our models. In addition to these, some physical and kinematical quantities have been generalized and discussed in Lyra and Riemann geometries. Also our results are consistent with the results of Yılmaz, Back et al. and Adcox et al. in four dimensions.
\end{abstract}

Keywords: Quark matter, FRW Universe, string cloud, higher dimension, Lyra geometry, domain wall.

\section{Introduction}

Type Ia Supernova observations demonstrated that our universe is accelerating [1], [2]. Physicists have proposed two probabilities to explain acceleration of the universe. One of the probability is phantom, k-essence, quintessence etc.[3] and the other is modified gravity theories[4]. Modified gravity theories have been studied for a long time by many researchers. These theories have been described as scalar field theory, scalar-tensor theory, vector-tensor theory and bimetric theory [5], [6]. Also, some of these theories are called Weyl theory[7], $\mathrm{f}(\mathrm{R})$ theory[8], Brans-Dicke theory[9], Yllmaz theory[10] and Lyra theory[11] [4]. According to Halford[12], in Lyra geometry, the constant displacement vector $\phi$ playing a role like constant cosmological constant $\Lambda$ in general relativity[13],[14]. In recent years most researchers have investigated Lyra geometry using different universe models with different energy-momentum tensors in four and five dimensions. For example, Singh investigated massive string with magnetic field in Lyra geometry for Bianchi V universe[15]. Mahanta and Mukherjee have studied some string models in Lyra manifold [16]. Aygün et al. have investigated non-existence of massive scalar field for the Marder universe in Lyra and Riemannian geometries[14]. The solutions of Einstein field equations in higher dimensions are important. Because we know that early phase of the universe is smaller than later phase [17]. Rahaman and Mandal have studied higher dimensional domain walls in Lyra geometry [18]. Yllmaz has obtained string cloud and domain walls with quark matter in 5-D Kaluza-Klein cosmological model [19]. Singh et.al. have examined effect of bulk viscous and G for five dimensional universe model in Lyra geometry [20]. Samanta and Debata have studied Bianchi I universe with strings in framework Lyra geometry [21]. Massive string cosmology has been investigated by Rahaman et al. in Lyra geometry for 5-D space-time model[22]. Rahaman et al. have obtained exact results for FRW metric in higher dimension Lyra geometry[23]. Katore et al.[24] have researched Bianchi I universe with mesonic stiff fluid models in Lyra's manifold. Yadav[25] studied inhomogeneous plane-symmetric models of perfect fluid in Lyra manifold with electro-magnetic field. Pradhan and Ram have researched plane symmetric magnetized inhomogeneous cosmological models in Lyra manifold[26]. Pradhan using $\Lambda$ term has investigated cylindrically symmetric universe with magnetized bulk viscous strings[27]. Also, who researched cylindrically symmetric viscous fluid universe in Lyra geometry[28]. Using constant deceleration parameter, Bianchi I string cosmological models in Lyra geometry have been studied by Pradhan and Singh[29]. However Agarwal et al.[30] have investigated massive strings in Lyra manifold for Bianchi type II universe. Bianchi type III cosmological model with strings has been researched by Pradhan et al. in Lyra geometry[31]. 
As mentioned above, various authors have investigated Lyra geometry using different matter forms and metrics in four and five dimensions. In this study, we have attached quark and strange quark matters to the string cloud and domain walls in Lyra geometry. This is reasonable because, approximately when the cosmic temperature was $\mathrm{T} \sim 200 \mathrm{MeV}$, it could have been such a phase transition. Quark Gluon Plasma(QGP) $\rightarrow$ hadron gas (called quark-hadron phase transition). [32],[33] [34]. In this topic, Bodmer[35], Witten[36] and Itoh[37] have declared two scenario for genesis of strange quark matter. First the quark-hadron phase transition in the early universe, the second conversion of neutron stars into SQM at ultrahigh densities[32],[33]-[38]. Generally, quark matter is formed with an equation of state (EoS) based on the factual bag model of quark matter. In the primitive version of the bag model, we suppose that the quarks are non interacting and massless[33]-[38]. In this case we have quark pressure

$$
p_{q}=\frac{\rho_{q}}{3}
$$

where $\rho_{q}$ is the quark energy density. The total energy density is

$$
\rho=\rho_{q}+B_{c}
$$

Also the total pressure is

$$
p=p_{q}-B_{c}
$$

and the equation of state for strange quark matter is given by

$$
p_{m}=\frac{1}{3}\left(p_{m}-4 B_{c}\right)
$$

where $B_{c}$ is the bag constant [32],[33]. Because quark-gluon plasma is created as a perfect liquid in the Brookhaven National Laboratory, we will take into account quark-gluon plasma in the form of perfect fluid following equation of state

$$
p_{m}=(\gamma-1) \rho_{m}
$$

where $1 \leq \gamma \preceq 2$ is a constant [19], [39], [40]. Recently, the solutions with strange quark matter were studied widely. Khadekar et al. have examined domain walls with strange quark matter for Kaluza-Klein type FRW universe [41]. Adhav et al. have studied Bianchi type III universe model with strange quark matter attached to cosmic string in GR[42]. Also, the study Bianchi III universe with strange quark matter attached to cosmic string have been studied by Mahanta et.al. in Self Creation cosmology[43]. Katore and Shaikh have researched strange quark matter attached to cosmic string for axially symmetric space-time in GR[44]. Yılmaz and Aktas have studied spherical symmetric space-time with strange quark matter attached to cosmic string in GR[45]. Solutions of N dimensional Kaluza-Klein universe with strange quark matter attached to cosmic string and domain wall have been obtained by Adhav et.al. in GR[33]. Pradhan et al. have investigated strange quark matter attached to cosmic string for spherical symmetric space-time considering with conformal motions[38].

In this paper, we have investigated strange quark matter attached to string cloud with vacuum solutions also domain walls with quark matter in higher dimensional flat FRW metric for Lyra and Riemann geometries. The paper is organized as follows: Modified Einstein field equations and its solutions with kinematical quantities for strange quark matter attached to string cloud in Lyra geometry are presented in Section 2. Domain walls with quark matter and strange quark matters in Lyra Geometry are presented in Section 3. The discussions of these models are given in Conclusion section.

\section{Strange Quark Matter Attached to String Cloud Solutions in Lyra Geometry}

The equation of Lyra geometry may be written as follows [46]

$$
R_{i k}-\frac{1}{2} g_{i k} R+\frac{3}{2} \phi_{i} \phi_{k}-\frac{3}{4} g_{i k} \phi_{j} \phi^{j}=-T_{i k}
$$


where we have assumed $8 \pi G=c=1$ also $\phi$ is the displacement vector and the other are components of the Riemannian geometry [47], [48]. Also, in this study for $(n+2)$ dimensional flat FRW metric, time-like displacement field vector is defined by [49]

$$
\phi_{i}=(\beta(t), 0,0,0 \ldots 0)
$$

Homogeneous, isotropic flat FRW universe is represented by;

$$
d s^{2}=-d t^{2}+R(t)^{2}\left[d r^{2}+r^{2} d x_{n}^{2}\right]
$$

and $R(t)$ represents the scale factor also[50]

$$
d x_{n}^{2}=d \theta_{1}^{2}+\sin ^{2} \theta_{1} d \theta_{2}^{2}+\ldots+\sin ^{2} \theta_{1} \sin ^{2} \theta_{2} \ldots \sin ^{2} \theta_{n-1} d \theta_{n}^{2}
$$

string cloud energy-momentum tensor is given by[51]

$$
T_{i k}=\rho u_{i} u_{k}-\rho_{s} X_{i} X_{k}
$$

where $\rho$ is the rest energy density for the cloud of strings with particles attached to them and $\rho_{s}$ is the string tension density and they are related by

$$
\rho=\rho_{p}+\rho_{s}
$$

where $\rho_{p}$ is the particle energy density. In this study we will take quarks instead of particles in the string cloud. Henceforth using eq.(11), we obtain

$$
\rho=\rho_{q}+\rho_{s}+B_{c}
$$

Using eqs. (10) and (12) we get new energy-momentum tensor for strange quark matter attached to string cloud as follows [32]

$$
T_{i k}=\left(\rho_{q}+\rho_{s}+B_{c}\right) u_{i} u_{k}-\rho_{s} X_{i} X_{k}
$$

where $u_{i}$ is the velocity of the particles and $X_{i}$ is the unit space like vector representing the direction of strings also

$$
u_{i} u^{i}=-X_{i} X^{i}=1 ; \quad u^{i} X_{i}=0
$$

Using eqs. (6),(8) and (13) we get field equations for $(n+2)$ dimensional flat FRW metric as follows

$$
\begin{aligned}
& \frac{n(n+1)}{2}\left(\frac{R_{t}^{2}}{R^{2}}\right)-\frac{3}{4} \beta^{2}=\rho_{q}+\rho_{s}+B_{c} \\
& \frac{n(n-1)}{2}\left(\frac{R_{t}^{2}}{R^{2}}\right)+n\left(\frac{R_{t, t}}{R}\right)+\frac{3}{4} \beta^{2}=\rho_{s} \\
& \frac{n(n-1)}{2}\left(\frac{R_{t}^{2}}{R^{2}}\right)+n\left(\frac{R_{t, t}}{R}\right)+\frac{3}{4} \beta^{2}=0
\end{aligned}
$$

here and thereafter the $t$ shows that differentiation with respect to cosmic time. In this paper we have three field equations with four unknowns $R, \beta^{2}, \rho_{s}$ and $\rho_{q}$. Also, the kinematical quantities such as velocity, cosmic expansion, Hubble parameter, spatial volume and deceleration parameter for $(n+2)$ dimensional generalized flat FRW universe are respectively given by

$$
\begin{gathered}
u_{i}=(-1,0,0 \ldots 0) \\
\theta=(n+1) \frac{R_{t}}{R}
\end{gathered}
$$




$$
\begin{gathered}
H=\frac{R_{t}}{R} \\
V=R^{(n+1)} r^{n}\left(\prod_{i=2}^{n} \sin ^{(i-1)} \theta_{n-i+1}\right) \\
q=-\frac{R R_{t, t}}{R_{t}^{2}}
\end{gathered}
$$

To solve field equations firstly, using the eqs. (16) and (17) we get string tension density is zero as follows

$$
\rho_{s}=0
$$

In this situation we have only two highly nonlinear field equations containing three unknowns, viz. $R(t), \rho_{q}$ and $\beta^{2}$. In order to obtain exact solutions, we use the conservation equation of the left and right sides of eq.(6), respectively;

$$
\left(R_{i}^{k}-\frac{1}{2} R g_{i}^{k}\right)_{; k}+\frac{3}{2}\left(\phi_{i} \phi^{k}\right)_{; k}-\frac{3}{4}\left(g_{i}^{k} \phi_{j} \phi^{j}\right)_{; k}=\frac{3}{2} \beta\left(\beta_{t}+(n+1) \frac{R_{t}}{R} \beta\right)
$$

and

$$
T_{i ; k}^{k}=0 ; \quad T_{i ; k}^{k}=\rho_{q, t}+(n+1) \frac{R_{t}}{R}\left(\rho_{q}+B_{c}\right)=0
$$

From eqs. (24) and (25) we obtain below quantities for $(n+2)$ dimensional FRW universe, respectively

$$
\begin{gathered}
\beta^{2}=\frac{c_{1}^{2}}{R^{(2 n+2)}} \\
R=\frac{c_{2}}{\left(\rho_{q}+B_{c}\right)^{\frac{1}{n+1}}}
\end{gathered}
$$

here $c_{1}$ and $c_{2}$ are the constants of integration. If we substitute these equations into the field eqs. (15) and(17), we get quark energy density as follows

$$
\rho_{q}=\frac{1}{\left(\frac{n+1}{2 n}\right)\left(t-c_{3}\right)^{2}-\frac{3}{4} \frac{c_{1}^{2}}{c_{2}^{(2 n+2)}}}-B_{c}
$$

here $c_{3}$ is the constant of integration. From eqs (28) and (27), we find $R(t)$ scale factor as follows

$$
R=c_{2}\left[\left(\frac{n+1}{2 n}\right)\left(t-c_{3}\right)^{2}-\frac{3}{4} \frac{c_{1}^{2}}{c_{2}^{2 n+2}}\right]^{\frac{1}{n+1}}
$$

From eqs. (26) and (29), we get $\beta^{2}$ function as follows

$$
\beta^{2}=\frac{c_{1}^{2}}{c_{2}^{2 n+2}\left(\frac{n+1}{2 n}\left(t-c_{3}\right)^{2}-\frac{3}{4} \frac{c_{1}^{2}}{c_{2}^{2 n+2}}\right)^{2}}
$$

Using eqs. (23) and (28) into the eq. (12), we get string energy density

$$
\rho=\frac{1}{\left(\frac{n+1}{2 n}\right)\left(t-c_{3}\right)^{2}-\frac{3}{4} \frac{c_{1}^{2}}{c_{2}^{(2 n+2)}}}
$$

From eqs.(11), (23) and (31) we get particle energy density $\rho_{p}=\rho-\rho_{s}$, like string energy density as follows

$$
\rho_{p}=\frac{1}{\left(\frac{n+1}{2 n}\right)\left(t-c_{3}\right)^{2}-\frac{3}{4} \frac{c_{1}^{2}}{c_{2}^{(2 n+2)}}}
$$


If we choose $\rho=\rho_{q}+\rho_{s}+B_{c}=0$ in eq. (12), we obtain the vacuum solution for the $(n+2)$ dimensional generalized flat FRW universe in Lyra geometry and we find scale factor $R(t)$ and $\beta^{2}$ function as follows

$$
\begin{gathered}
R_{\text {vacuum }}=\left[(n+1)\left(c_{4} t+c_{5}\right)\right]^{\frac{1}{n+1}} \\
\beta_{\text {vacuum }}^{2}=\frac{2 n}{3(n+1)} \frac{c_{1}^{2}}{\left(c_{4} t+c_{5}\right)^{2}}
\end{gathered}
$$

here $c_{4}$ and $c_{5}$ are arbitrary integration constants.

\section{Domain Walls with Quark Matter in Lyra Geometry}

The energy-momentum tensor of a domain wall in the classical form is given by

$$
T_{i k}^{D}=(\rho+p) u_{i} u_{k}+p g_{i k}
$$

This perfect-fluid form of the domain wall includes quark matter.(described by $\rho_{m}=\rho_{q}+B_{c}$ and $\left.p_{m}=p_{q}-B_{c}\right)$ as well as domain wall tension $\sigma_{w}$; i.e. $\rho=\rho_{m}+\sigma_{w}$ and $p=p_{m}-\sigma_{w}$. Also $p_{m}$ and $\rho_{m}$ are related by the bag model equation of state, i.e. eq. (4) and equation of state, i.e. eq. (5), [19], [41]. Using the eqs. (6), (8) and (35) the field equations we get field equations for $(n+2)$ dimensional flat FRW metric for domain walls with quark matter as follows

$$
\begin{gathered}
\frac{n(n+1)}{2} \frac{R_{t}^{2}}{R^{2}}-\frac{3}{4} \beta^{2}=\rho \\
\frac{n R_{t, t}}{R}+\frac{n(n-1)}{2} \frac{R_{t}^{2}}{R^{2}}+\frac{3}{4} \beta^{2}=-p
\end{gathered}
$$

In this section there are two independent field equations with four unknowns $R, \rho, p$ and $\beta^{2}$. To solve the system completely we need two approximations. One of these considerations is conservation equation for this model;

$$
\frac{3}{2} \beta\left(\beta_{t}+(n+1) \frac{R_{t}}{R} \beta\right)=0
$$

If we solve eq. (38), we find

$$
\beta^{2}=\frac{k_{1}^{2}}{R^{2 n+2}}
$$

here $k_{1}$ is an constant. The other consideration is varying deceleration parameter. Because deceleration parameter $(q)$ and its sign are so important parameter to determine the fate of the universe. Also $q$ indicates whether the model accelerates or not [52], [53] and given by

$$
q=-\frac{R R_{t, t}}{R_{t}^{2}}=m-1
$$

here $\mathrm{m}$ is a constant. If we solve eq.(40), we get two solutions as follows

$$
\begin{aligned}
& R=s_{2} e^{s_{1} t} \quad \text { for } m=0 \\
& R=\left[m\left(k_{2} t+k_{3}\right)\right]^{\frac{1}{m}} \quad \text { for } \quad m \neq 0
\end{aligned}
$$

where $s_{1}, s_{2}, k_{2}$ and $k_{3}$ are constants. In this study we will investigate for $m \neq 0$ situation in higher dimensional FRW universe. From eqs. (39) and (42) we find the displacement vector field as follows

$$
\beta^{2}=\frac{k_{1}^{2}}{\left(m\left(k_{2} t+k_{3}\right)\right)^{\frac{2 n+2}{m}}}
$$


From eqs. (36), (42) and (43) we obtain domain wall density as follows

$$
\rho=\rho_{m}+\sigma_{w}=\frac{n(n+1) k_{2}^{2}}{2 m^{2}\left(k_{2} t+k_{3}\right)^{2}}-\frac{3 k_{1}^{2}}{4\left(m\left(k_{2} t+k_{3}\right)\right)^{\frac{2 n+2}{m}}}
$$

From eqs. (37), (42) and (43) we get domain wall pressure as follows

$$
p=p_{m}-\sigma_{w}=\frac{n k_{2}^{2}(2 m-n-1)}{2 m^{2}\left(k_{2} t+k_{3}\right)^{2}}-\frac{3 k_{1}^{2}}{4\left(m\left(k_{2} t+k_{3}\right)\right)^{\frac{2 n+2}{m}}}
$$

\subsection{Case (i) Domain Walls with Strange Quark Matter in Lyra Geometry}

If we use eq. (4) in eqs. (44) and (45), i.e. strange quark matter coupled to the domain walls, we get

$$
\begin{gathered}
\rho_{q}=\frac{3 n k_{2}^{2}}{4 m\left(k_{2} t+k_{3}\right)^{2}}-\frac{9 k_{1}^{2}}{8\left(m\left(k_{2} t+k_{3}\right)\right)^{\frac{2 n+2}{m}}} \\
p_{q}=\frac{n k_{2}^{2}}{4 m\left(k_{2} t+k_{3}\right)^{2}}-\frac{3 k_{1}^{2}}{8\left(m\left(k_{2} t+k_{3}\right)\right)^{\frac{2 n+2}{m}}} \\
\sigma_{w}=\frac{3 k_{1}^{2}}{8\left(m\left(k_{2} t+k_{3}\right)^{\frac{2 n+2}{m}}\right.}-\frac{n k_{2}^{2}(3 m-2 n-2)}{4 m^{2}\left(k_{2} t+k_{3}\right)^{2}}-B_{c}
\end{gathered}
$$

\subsection{Case (ii) Domain Walls with Quark Matter in Lyra Geometry}

If we use eq. (5) in eqs. (44) and (45), i.e. quark matter coupled to the domain walls, we get

$$
\begin{gathered}
\rho_{m}=\frac{1}{2 \gamma}\left(\frac{2 n k_{2}^{2}}{m\left(k_{2} t+k_{3}\right)^{2}}-\frac{3 k_{1}^{2}}{\left(m\left(k_{2} t+k_{3}\right)\right)^{\frac{2 n+2}{m}}}\right) \\
p_{m}=\frac{\gamma-1}{2 \gamma}\left(\frac{2 n k_{2}^{2}}{m\left(k_{2} t+k_{3}\right)^{2}}-\frac{3 k_{1}^{2}}{\left(m\left(k_{2} t+k_{3}\right)\right)^{\frac{2 n+2}{m}}}\right) \\
\sigma_{\omega}=\frac{1}{4 \gamma}\left(\frac{2 n k_{2}^{2}(\gamma(n+1)-2 m)}{m^{2}\left(k_{2} t+k_{3}\right)^{2}}+\frac{k_{1}^{2}(6-3 \gamma)}{\left(m\left(k_{2} t+k_{3}\right)\right)^{\frac{2 n+2}{m}}}\right)
\end{gathered}
$$

\section{Conclusions}

In this paper we have obtained exact solutions of strange quark matter attached to string cloud and domain walls in $(n+2)$ dimensional flat FRW type cosmological model. From obtained solutions we have following properties:

A) In this paper firstly we have obtained exact solutions for the generalized higher dimensional flat FRW universe in the presence of strange quark matter attached to string cloud also vacuum situations in Lyra and Riemann geometries. According to Letelier; [54] there are two fundamental subjects to study string clouds. Firstly; for many interactions to construct good models, at a classical level the relativistic strings can be used [55], [56]. Secondly, the universe can be symbolized as a gathering of extended objects [54]. Also, cosmic strings have significant role and could give the information about evolution of the early universe before particle creation [57]. Many researchers have studied cosmic strings in general relativity and various alternative gravitation theories. Kiran and Reddy have researched Bianchi type III bulk viscous strings in $f(R, T)$ gravity and they found that $\rho_{s}=0$ also they said that "Bianchi type-III bulk viscous string cosmological model does not survive in this theory" [58]. In Bimetric theory Reddy investigated cosmic strings using Bianchi I metric and found $\rho_{s}=0$ and discussed that "cosmic strings do not occur in Bimetric theory of gravitation in Bianchi I metric". Also Krori et al. [59] studied Bianchi V metric and found $\rho_{s}=0$ in general relativity [60]. In Bimetric theory Sahoo and Mishra have studied 
domain walls and string cloud with quark matter[61] also strange quark matter attached to string cloud [62]. They obtained that string density is zero $\left(\rho_{s}=0\right)$ in these studies. According to Sahoo and Mishra "there is no contribution from strings for these models in bimetric theory" [61], [62]. However Mohanty et al. investigated string cosmology in Lyra and Riemann geometries in five dimensions and they found that "cosmic string models do not survive in both the theories" i.e. $\rho_{s}=0$ [63].

Similar to other studies in this paper we have obtained string tension density is zero everywhere $\left(\rho_{s}=0\right)$ also we could say that there is no contribution from strings for $(n+2)$ dimensional flat FRW universe with attached strange quark matter in Lyra and Riemann geometries. But we know that cosmic strings play an important role in the formation of the galaxies[57]. Then it may be said that our model does not help to define the early era of the universe therefore the matter contribution come from the quark energy density in higher dimensional Lyra and Riemann geometries. From Eqs. (8) and (29), we can rewrite the line element in the Lyra geometry following form

$$
d s^{2}=-d t^{2}+\left(c_{2}\left[\left(\frac{n+1}{2 n}\right)\left(t-c_{3}\right)^{2}-\frac{3}{4} \frac{c_{1}^{2}}{c_{2}^{2 n+2}}\right]^{\frac{1}{n+1}}\right)^{2}\left[d r^{2}+r^{2} d x_{n}^{2}\right]
$$

here $d x_{n}^{2}$ is also same to eq.(9). From the results of Eqs. (26) and (27), we see that $c_{2}$ is an important non-zero constant $c_{2} \neq 0$ in Lyra and Riemann geometries. However, using eqs. (18)-(22) and (27), we get the kinematical quantities such as velocity, cosmic expansion, Hubble parameter, spatial volume and deceleration parameter for $(n+2)$ dimensional generalized flat FRW universe in Lyra geometry as follows,

$$
\begin{gathered}
u_{i}=(-1,0,0 \ldots 0) \\
\theta=\frac{2\left(t-c_{3}\right)}{\left(t-c_{3}\right)^{2}-\frac{3 n}{2 n+2}\left(\frac{c_{1}^{2}}{c_{2}^{2 n+2}}\right)} \\
H=\frac{1}{n} \frac{t-c_{3}}{\frac{n+1}{2 n}\left(t-c_{3}\right)^{2}-\frac{3 c_{1}^{2}}{4 c_{2}^{2 n+2}}} \\
V=c_{2}^{n+1}\left[\frac{n+1}{2 n}\left(t-c_{3}\right)^{2}-\frac{3}{4} \frac{c_{1}^{2}}{c_{2}^{2 n+2}}\right] r^{n}\left(\prod_{i=2}^{n}\left(\sin \left(\theta_{n-i+1}\right)\right)^{i-1}\right) \\
q=\frac{n-1}{2}+\frac{3 c_{1}^{2}}{2\left(t-c_{3}\right)^{2} c_{2}^{2 n+2}}
\end{gathered}
$$

At the initial stage of the universe, when $t \rightarrow 0$, the scale factor $\mathrm{R}(\mathrm{t})$, quark pressure, particle energy density, total pressure, displacement field $\beta^{2}$ and the kinematical quantities are constants in Lyra geometry and also $R_{\text {vacuum }}, \beta_{\text {vacuum }}^{2}$ are constants in vacuum model.

When $\mathrm{t}$ increases the scale factor and spatial volume increase but quark pressure, quark density, Hubble parameter $(\mathrm{H})$, expansion scalar $(\theta)$ and deceleration parameter $(\mathrm{q})$ and displacement field vector $\beta^{2}$ decrease in Lyra geometry. These results agree with Halford's study [12]. According to Halford [12] the displacement vector field plays role of cosmological constant in Lyra geometry [29]. Also, recent cosmological observations of SNe Ia show that the value of the cosmological constant is a positive value $\approx 10^{-123}[64],[65],[66],[29]$.

If we take $c_{1}=0$ in Eq.(26), we get $\beta^{2}=0$. Using this equality in Eqs.(28)-(32), we get the results of higher dimensional generalized flat FRW universe with strange quark matter attached to string cloud in Riemann geometry as follows

Scale factor

$$
R=c_{2}\left[\left(\frac{n+1}{2 n}\right)\left(t-c_{3}\right)^{2}\right]^{\frac{1}{n+1}}
$$

Quark energy density 


$$
\rho_{q}=\frac{2 n}{(n+1)} \frac{1}{\left(t-c_{3}\right)^{2}}-B_{c}
$$

String tension density

$$
\rho_{s}=0
$$

String energy density

$$
\rho=\frac{2 n}{(n+1)} \frac{1}{\left(t-c_{3}\right)^{2}}
$$

String particle density $\left(\rho_{p}=\rho-\rho_{s}\right)$

$$
\rho_{p}=\frac{2 n}{(n+1)} \frac{1}{\left(t-c_{3}\right)^{2}}
$$

From Eqs. (8) and (58), we can rewrite the line element in the Riemann geometry following form

$$
d s^{2}=-d t^{2}+c_{2}^{2}\left[\frac{n+1}{2 n}\left(t-c_{3}\right)^{2}\right]^{\frac{2}{n+1}}\left[d r^{2}+r^{2} d x_{n}^{2}\right]
$$

Substituting eq. (58) into the eqs. (19)-(22), we obtain the kinematical quantities such as velocity, cosmic expansion, Hubble parameter, spatial volume and deceleration parameter for $(n+2)$ dimensional generalized flat FRW universe in Riemann geometry as follows,

$$
\begin{gathered}
u_{i}=(-1,0,0 \ldots 0) \\
\theta=\frac{2}{\left(t-c_{3}\right)} \\
H=\frac{2}{(n+1)\left(t-c_{3}\right)} \\
V=c_{2}^{n+1}\left[\frac{n+1}{2 n}\left(t-c_{3}\right)^{2}\right] r^{n}\left(\prod_{i=2}^{n}\left(\sin \left(\theta_{n-i+1}\right)\right)^{i-1}\right) \\
q=\frac{n-1}{2}
\end{gathered}
$$

At the initial stage of the universe, when $t \rightarrow 0$, the scale factor $\mathrm{R}(\mathrm{t})$, quark pressure, particle energy density, quark density, total pressure and the kinematical quantities are constants in Riemann geometry and also $R_{\text {vacuum }}$ is a constant in vacuum model. When t increases the scale factor and spatial volume increase but quark pressure, quark density, total pressure, string particle density, Hubble parameter $(\mathrm{H})$, expansion scalar $(\theta)$ decrease. These results are consistent with the results of Yilmaz[19], Back et al.[39], Adcox et al[40] at Brookhaven National Laboratory and deceleration parameter $q$ is a constant in Riemann geometry, this shows that our model decelerates in the standard way [47]. Also we obtain strange quark matter attached string cloud in four-dimensional FRW solutions for $n=2$ in Lyra and Riemann geometries.

B) In this part we have obtained exact solutions for the generalized higher dimensional flat FRW universe in the presence of domain wall includes quark matter. From Eqs. (8) and (42), we can rewrite FRW line element for domain wall includes quark and strange quark matter in Lyra manifold

$$
d s^{2}=-d t^{2}+\left[m\left(k_{2} t+k_{3}\right)\right]^{\frac{2}{m}}\left[d r^{2}+r^{2} d x_{n}^{2}\right]
$$

At the initial stage of the universe, when $t \rightarrow 0$, the scale factor $\mathrm{R}(\mathrm{t})$, domain wall density $\rho=\rho_{m}+\sigma_{w}$ and pressure $p=p_{m}-\sigma_{w}$, displacement field vector $\beta^{2}$ are constants for domain wall solutions in Lyra 
geometry. Also $\rho_{q}, p_{q}$ and $\sigma_{w}$ are constants for domain wall includes strange quark matter and $\rho_{m}, p_{m}$ and $\sigma_{w}$ are constants for domain wall includes quark matter in Lyra geometry. When t increases the scale factor $\mathrm{R}(\mathrm{t})$ increases but domain wall density $\rho=\rho_{m}+\sigma_{w}$ and pressure $p=p_{m}-\sigma_{w}$, displacement field vector $\beta^{2}$ decrease. Also $\rho_{q}, p_{q}$ and $\sigma_{w}$ decrease for domain wall that includes strange quark matter and $\rho_{m}, p_{m}$ and $\sigma_{w}$ increase for domain wall that includes quark matter in Lyra geometry. According to Halford [12] the displacement vector field plays role of cosmological constant in Lyra geometry [29]. Also, recent cosmological observations of SNe Ia show that the value of the cosmological constant is a positive value $\approx 10^{-123}$ [64], [65], [66],[29]. In this context, our results agree with Halford's study [12] as string cloud results. In these results $k_{1}$ is an important constant. If we take $k_{1}=0$ in eq.(43), we get $\beta^{2}=0$. Using this equality in eqs.(44)-(51) we get the density and pressure of the domain wall in higher dimensional generalized flat FRW universe with domain wall in Riemann geometry as follows;

$$
\begin{gathered}
\rho=\rho_{m}+\sigma_{w}=\frac{n(n+1) k_{2}^{2}}{2 m^{2}\left(k_{2} t+k_{3}\right)^{2}} \\
p=p_{m}-\sigma_{w}=\frac{n k_{2}^{2}(2 m-n-1)}{2 m^{2}\left(k_{2} t+k_{3}\right)^{2}}
\end{gathered}
$$

The density and pressure of strange quark matter coupled to the domain walls with domain wall tension in Riemann geometry are given by

$$
\begin{gathered}
\rho_{q}=\frac{3 n k_{2}^{2}}{4 m\left(k_{2} t+k_{3}\right)^{2}} \\
p_{q}=\frac{n k_{2}^{2}}{4 m\left(k_{2} t+k_{3}\right)^{2}} \\
\sigma_{w}=\frac{n k_{2}^{2}(2 n+2-3 m)}{4 m^{2}\left(k_{2} t+k_{3}\right)^{2}}-B_{c}
\end{gathered}
$$

The density and pressure of quark matter coupled to the domain walls with domain wall tension in Riemann geometry are given by

$$
\begin{gathered}
\rho_{m}=\frac{1}{\gamma}\left(\frac{n k_{2}^{2}}{m\left(k_{2} t+k_{3}\right)^{2}}\right) \\
p_{m}=\frac{\gamma-1}{\gamma}\left(\frac{n k_{2}^{2}}{m\left(k_{2} t+k_{3}\right)^{2}}\right) \\
\sigma_{\omega}=\frac{1}{2 \gamma}\left(\frac{n k_{2}^{2}(\gamma(n+1)-2 m)}{m^{2}\left(k_{2} t+k_{3}\right)^{2}}\right)
\end{gathered}
$$

In the case of quark matter coupled to domain walls (i.e. Case ii), if we take $\gamma=1$ in eqs.(49)-(50) and (75)-(76), we get domain wall solutions with dust quark matter. When $\gamma=\frac{4}{3}$ we have domain wall solutions with quark matter like radiation. When $\gamma=2$ we have stiff quark matter solution in Lyra and Riemann geometries. Also, for $n=2$ we obtain domain wall and includes quark matter domain wall solutions for four-dimensional flat FRW solutions in Lyra and Riemann geometries.

Acknowledgement We thank for anonymous referee careful reading of the manuscript and suggestions. This work was supported by Çanakkale Onsekiz Mart University Scientific Research Coordination Unit. Project number:FDK-2016-784. 


\section{References}

1. M. Tegmark, et al.,"Cosmological parameters from SDSS and WMAP," Phys. Rev. D, vol. 69, 103501-103528, 2004.

2. G. Efstathiou, et al.,"Evidence for a non-zero $\Lambda$ and a low matter density from a combined analysis of the $2 \mathrm{dF}$ Galaxy Redshift Survey and cosmic microwave background anisotropies," Mon. Not. Roy. Astron. Soc., vol. 330, pp. L29-L35, 2002.

3. N. Bilic, "Thermodynamics of Dark Energy," arXiv:0812.5050v2.

4. C. Aktaș, S. Aygün, İ. Yılmaz, "Behaviors of dark energy and mesonic scalar field for anisotropic universe in f(R) gravity," Physics Letters B, vol. 707, 237-242, 2012.

5. B. M. Barker, "General scalar-tensor theory of gravity with constant G," Astrophysical Journal vol. 219, pp. 5-11, 1978.

6. J. D. Bekenstein, "Relativistic gravitation theory for the modified Newtonian dynamics paradigm," Phys. Rev. $D$, vol. 70, no. 8, pp. 083509-083543, 2004.

7. H. Weyl," Gravitation und Elektrizitat," in Preuss akad. Wissi Berlin, pp. 465-478, 1918.

8. F. S. N. Lobo and M. A. Oliveira, "Wormhole geometries in $\mathrm{f}(\mathrm{R})$ modified theories of gravity" Phys. Rev. D., vol. 80, no. 10, pp. 104012-104020, 2009.

9. C. Brans and R. H. Dicke, "Mach's Principle and a Relativistic Theory of Gravitation*," Phys. Rev. vol. 124, no. 3, pp. 925-935, 1961.

10. H. Yılmaz, "New Approach to General Relativity," Phys. Rev., vol. 111, no. 5, pp. 1417-1426, 1958.

11. G. Lyra, "Über eine Modifikation der Riemannschen Geometrie," Mathematische Zeitschrift, vol 54, no. 1, pp. 52-64, 1951

12. W. D. Halford, "Cosmological theory based on Lyra's geometry," Australian Journal of Physics, vol. 23, pp. 863-869, 1970.

13. A. Beesham, "Vacuum Friedmann cosmology based on Lyra's manifold," Astrophys. Space Sci., vol. 127, pp. 189-191, 1986.

14. S. Aygün, C. Aktaș and İ. Yılmaz, "Non-existence of a massive scalar field for the Marder universe in Lyra and Riemannian geometries," Journal of Geometry and Physics, vol. 62, no. 1, pp. 100-106, 2012.

15. J. K. Singh, "String Cosmological Models in Lyra Geometry," Int. J. Theor. Phys., vol. 48, no. 3, pp. 905-912, 2009.

16. P. Mahanta and A. Mukherjee, "String models in Lyra geometry," Indian J. Püre appl. Math. 32,199 (2001).

17. A. Chodos and S. Detweller, "Where has the fifth dimension gone?," Physical Review D, vol. 21, no. 8, pp. 2167-2170, 1980.

18. F. Rahaman and S. Mandal, "Gravitational field of higher dimensional domain walls in Lyra geometry," arXiv:gr-qc/0608082.

19. İ. Yllmaz, " String cloud and domain walls with quark matter in 5-D Kaluza Klein cosmological model," General Relativity and Gravitation, vol. 38, no. 9, pp. 1397-1406, 2006.

20. G. P. Singh, R. V. Deshpande and T. Singh, "Higher-dimensional cosmological model with variable gravitational constant and bulk viscosity in Lyra geometry," Pramana, vol. 63, no. 5, pp. 937-945. 2004.

21. G. C. Samanta and S. Debata, Journal of Mod. Phys. 3, 180 (2012).

22. F. Rahaman, S. Chakraborty, S. Das, M. Hossain and J. Bera, "Higher-dimensional string theory in Lyra geometry," Pramana, vol. 60, no. 3, pp. 453-459, 2003.

23. F. Rahaman, S. Das, N. Begum, M. Hossain, "Higher dimensional homogeneous cosmology in Lyra geometry," Pramana, vol. 61, no. 1, pp. 153-159 2003.

24. S. D. Katore, S. V. Thakare and K. S. Adhav, "Bianchi Type-I cosmological mesonic stiff fluid models in Lyra's geometry,", Pramana, vol. 71, no. 1, pp. 15-22, 2008.

25. A. K. Yadav, "Lyra's cosmology of inhomogeneous universe with electromagnetic field," Fizika B, vol. 19, pp. 53-80, 2010.

26. A. Pradhan and P. Ram, "A Plane-Symmetric Magnetized Inhomogeneous Cosmological Models of Perfect Fluid Distribution with Variable Magnetic Permeability in Lyra Geometry," Int. J. Theor. Phys., vol. 48, pp. 3188-3201, 2009.

27. A. Pradhan, "Some Magnetized Bulk Viscous String Cosmological Models in Cylindrically Symmetric Inhomogeneous Universe with Variable A-Term," Commun. Theor. Phys., vol. 51, pp. 367-374, 2009.

28. A. Pradhan, "Cylindrically symmetric viscous fluid universe in Lyra geometry," Jour. Math. Phys., vol. 50, pp. 022501-022512, 2009.

29. A. Pradhan and A. K. Singh, "Anisotropic Bianchi Type-I String Cosmological Models in Normal Gauge for Lyra's Manifold with Constant Deceleration Parameter," Int. J. of Theor. Phys., vol. 50, no. 3 pp. 916-913, 2011. 30. S. Agarwal, R. K. Pandey and A. Pradhan, "Bianchi type-II string cosmological models in normal gauge for Lyra's manifold with constant deceleration parameter," Indian J. Phys., vol. 86, no. 1, pp. 61-70, 2012. 
31. A. Pradhan, H. Amirhashchi and H. Zainuddin, "Exact solution of perfect fluid massive string cosmology in Bianchi type III space-time with decaying vacuum energy density 4 ," Astrophy. and Space Sci., vol. 331, no. 2, pp. 679-687, 2011.

32. İ. Yavuz, İ. Yılmaz and H. Baysal, "Strange Quark Matter Attached to the String Cloud in the Spherical Symmetric Space-Time Admitting Conformal Motion," Int. J. Mod. Phys. D, vol. 14, no. 08, pp. 1365-1372, 2005 .

33. K. S. Adhav, A. S. Nimkar, M. V. Dawande, "String Cloud and Domain Walls with Quark Matter in n-Dimensional Kaluza-Klein Cosmological Model," Int. J. Theor. Phys., vol. 47, no. 7, pp. 2002-2010, 2008.

34. H. Cağlar and S. Aygün, "Non-existence of Brans-Dicke theory in higher dimensional FRW universe," Astrophys. and Space Sci., vol. 361, no. 6, pp. 200-2005, 2016.

35. A. R. Bodmer, "Collapsed Nuclei," Phys. Rev. D, vol. 4, no. 6, pp. 1601-1606, 1971.

36. E. Witten, "Cosmic separation of phases," Phys. Rev. D , vol. 30, no. 2, pp. 272-285, 1984.

37. N. Itoh, "Hydrostatic Equilibrium of Hypothetical Quark Stars," Prog. Theor. Phys., vol. 44, no. 1, pp. 291-292, 1970 .

38. A. Pradhan, G. S. Khadekar, M. M. Kumar and S. Kumbhare, "Higher Dimensional Strange Quark Matter Coupled to the String Cloud with Electromagnetic Field Admitting One Parameter Group of Conformal Motion," Chin. Phys. Lett., vol. 24, no. 10, pp. 3013-3016, 2007.

39. B. B. Back, et al., "The PHOBOS perspective on discoveries at RHIC," Nucl Phys. A, vol 757, no. 1, pp. 28-101, 2005.

40. K. Adcox, et al., "Formation of dense partonic matter in relativistic nucleus-nucleus collisions at RHIC: Experimental evaluation by the PHENIX Collaboration," Nucl Phys. A, vol. 757, no. 1, pp. 184-283, 2005.

41. G. S. Khadekar, R. Wanjari and C. Ozel, "Domain Wall with Strange Quark Matter in Kaluza-Klein Type Cosmological Model," Int. J. Theor. Phys., vol. 48, no. 9, pp. 2550-2557, 2009.

42. K. S. Adhav, A. S. Nimkar, V. B. Raut and R. S. Thakare, "Strange quark matter attached to string cloud in Bianchi type-III space time," Astrophys. Space Sci., vol. 319, no. 1, pp. 81-84, 2009.

43. K. L. Mahanta, S. V. Biswal, P. K. Sahoo and M. C. Adhikary, "String Cloud with Quark Matter in Self-creation Cosmology," Int. J. Theor. Phys., vol. 51, no. 5, pp. 1538-1544, 2012.

44. S. D. Katore and A. Y. Shaikh, "Cosmological Model with Strange Quark Matter Attached to Cosmic String for Axially Symmetric Space-Time," Int. J. Theor. Phys., vol. 51, no. 6, pp. 1881-1888, 2012.

45. İ. Yılmaz and C. Aktas,, "Space-Time Geometry of Quark and Strange Quark Matter," Chin. J. Astron. Astrophys., vol. 7, no. 6, pp. 757-763, 2007.

46. D. K. Sen and K. A. Dunn, "A Scalar-Tensor Theory of Gravitation in a Modified Riemannian Manifold," J. Math. Phys., vol. 12, no. 4, pp. 578-586, 1971.

47. D. R. K. Reddy, "Plane Symmetric Cosmic Strings In Lyra Manifold," Astrophys. Space Sci., vol. 300, no. 4, pp. 381-386, 2005.

48. F. Rahaman, S. Chakraborty, N. Begum, M. Hossain, and M. Kalam, "Bianchi-IX string cosmological model in Lyra geometry," Pramana J. Phys., vol. 60, no.6, pp. 1153-1159, 2003.

49. G. P. Singh, S. Kotambkar and A. Pradhan, "Higher Dimensional Cosmological Model in Lyra Geometry," Int. J. Mod. Phys. D., vol. 12, no. 05, pp. 853-860, 2003.

50. C. P. Singh and A. Beesham, "Particle Creation in Higher Dimensional Space-time with Variable G and $\Lambda$," Int. J. Theor. Phys., vol. 51, no. 12, pp. 3951-3962, 2012.

51. P. S. Letelier, "String cosmologies," Phys. Rev. D, vol. 28, no. 10, pp. 2414-2419, 1983.

52. K. S. Adhav, "LRS Bianchi type-I cosmological model with linearly varying deceleration parameter," Eur. Phys. J. Plus, vol. 126, pp. 122-126, 2011.

53. Ö. Akarsu, T. Dereli, "Cosmological Models with Linearly Varying Deceleration Parameter," Int. J. Theor. Phys., vol. 51, no. 2, pp. 612-621, 2012.

54. P. S. Letelier, "Clouds of strings in general relativity," Physical Review D, vol. 20, no. 6, pp. 1294-1302, 1979. 55. M. Kalb, P. Ramond, "Classical direct interstring action," Phys. Rev. D 9, 2273 (1974). ; M. Kalb, ibid. 17, 2713 (1978).

56. P. S. Letelier, "Gauge-invariant theory of direct interaction between strings," Phys. Rev. D, vol. 15, no. 4, pp. 1055-1062, 1977.

57. K. S. Adhav, P. S. Gadodia snd A. S. Bansod, "Bianchi Type-I String Cosmological Model in Creation-Field," Int. J. of Theo. Phys., vol. 50, no. 9, pp. 2720-2736, 2009.

58. M. Kiran and D. R. K. Reddy, "Non-existence of Bianchi type-III bulk viscous string cosmological model in $\mathrm{f}($ R, T) gravity," Astrophys Space Sci., vol. 346, no. 2, pp. 521-524, 2013.

59. K. D. Krori, T. Chaudhury and C. R. Mahanta, "Strings in some Bianchi type cosmologies," Gen. Rel. and Grav., vol. 26, no. 3, pp. 265-274, 1994.

60. D. R. K. Reddy, "Non-existence of cosmic strings in bimetric theory of gravitation," Astrophys. and Space Sci., vol. 286, no. 3, pp. 397-400, 2003. 
61. P. K. Sahoo, B. Mishra, "String cloud and domain walls with quark matter for plane symmetric cosmological model in bimetric theory," Journal of Theoretical and Applied Physics vol. 7, no. 1, pp. 12-16, 2013.

62. P.K. Sahoo, B. Mishra, "Axially symmetric space-time with strange quark matter attached to string cloud in bimetric theory," Int. J.Pure and Applied Math. 82, 87 (2013).

63. G. Mohanty, R. R. Sahoo and B. K. Bishi, "Non-existence of five dimensional string cosmological models in Riemannian and Lyra geometries," Astrophys. Space Sci., vol. 319, no. 1, pp. 75-79, 2009.

64. P. M. Garnavich, et al., "Supernova Limits on the Cosmic Equation of State," Astrophys. J., vol. 509, no. 1, pp. 74-79, 1998.

65. S. Perlmutter, et al., "Measurements of the Cosmological Parameters $\Omega$ and $\Lambda$ from the First Seven Supernovae at $z>0.35$," Astrophys. J., vol. 483, no. 2, pp. 565-581, 1997. 0.35," Astrophys. J., vol. 483, no. 2, pp. 565-581, 1997.

66. B. P. Schmidt, "The High-Z Supernova Search: Measuring Cosmic Deceleration and Global Curvature of the Universe Using Type IA Supernovae" Astrophys J., vol. 507, no. 1, pp. 46-63, 1998. 